\title{
How This Book was Created Using Collaborative Authoring and Cloud Tools
}

\author{
Sönke Bartling
}

\begin{abstract}
This book about novel publishing and collaboration methods of scholarly knowledge was itself created using novel and collaborative authoring tools. Google Docs as a collaborative authoring and text editing tool and Dropbox as a cloud storage solution were used. Our experience was a positive one and we think that it saved us a lot of organisational emails and hundreds of work hours. Here we describe the workflow process in detail so that the interested author might benefit from what we learnt.
\end{abstract}

\section{How this Book was Created}

The creation process can be divided in several phases in regard to the online tools which were used.

Phase I: Potential content was collected and authors were invited to participate. Shortly afterwards, a table in Google Docs was collaboratively maintained by both editors. For each chapter, the title and possible authors were discussed, emails to authors were sent, and feedback was added. Chapters were divided among both editors, so that one contact person was responsible for each chapter. In jour-fixe Skype sessions the status of the work in progress was discussed.

Phase II: A table of contents was created as a text document in Google Docs. The returning abstracts were uploaded to Google Docs and the links were created to the abstracts. The table of contents file served as the central document (Fig. 1).

\author{
S. Bartling $(\bowtie)$ \\ German Cancer Research Center, Heidelberg, Germany \\ e-mail: soenkebartling@gmx.de \\ S. Bartling \\ Institute for Clinical Radiology and Nuclear Medicine, Mannheim University Medical \\ Center, Heidelberg University, Mannheim, Germany
}




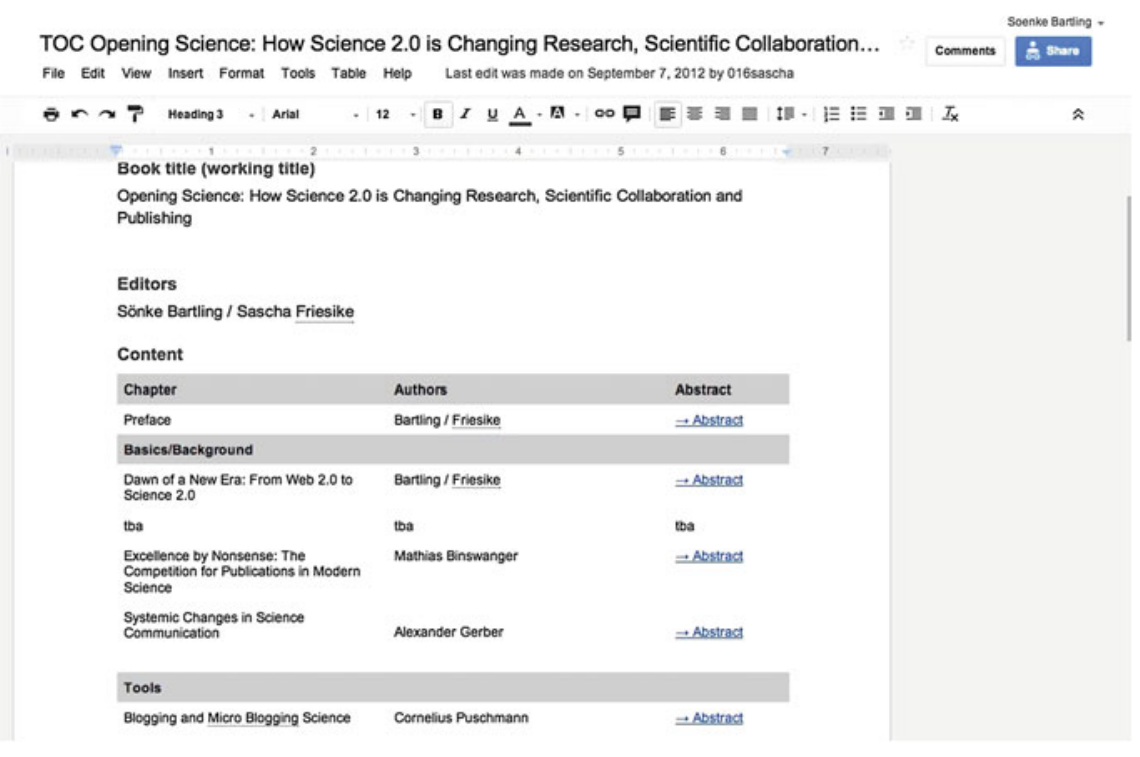

Fig. 1 The table of contents was the central workplace for the editors in the early phase of this book project

Phase III: Returning articles were uploaded to Google Docs and the authors were invited to participate with 'editing' privileges (Fig. 2). Articles were also linked to the TOC. Authors and editors worked iteratively on the Google documents. Commenting functionality was used to discuss points of controversy. Images were designed in Apple Keynote and the image files and other files were shared using Dropbox.

Phase IV: An internal review started once almost final versions of the chapters existed. All authors received a TOC with links to every chapter-all authors possessed commenting privileges for all other chapters. Only the chapter authors and editors had the right to change text. The internal references within the book were set in this phase and consistency among the chapters was assured. Citations were added using the Harvard author-date style, omitting the necessity of changing in-text references if novel references were added. Since Google Docs lacks integration with a reference management system, Zotero was used to import the references from databases. The bibliographies for each chapter were generated from 


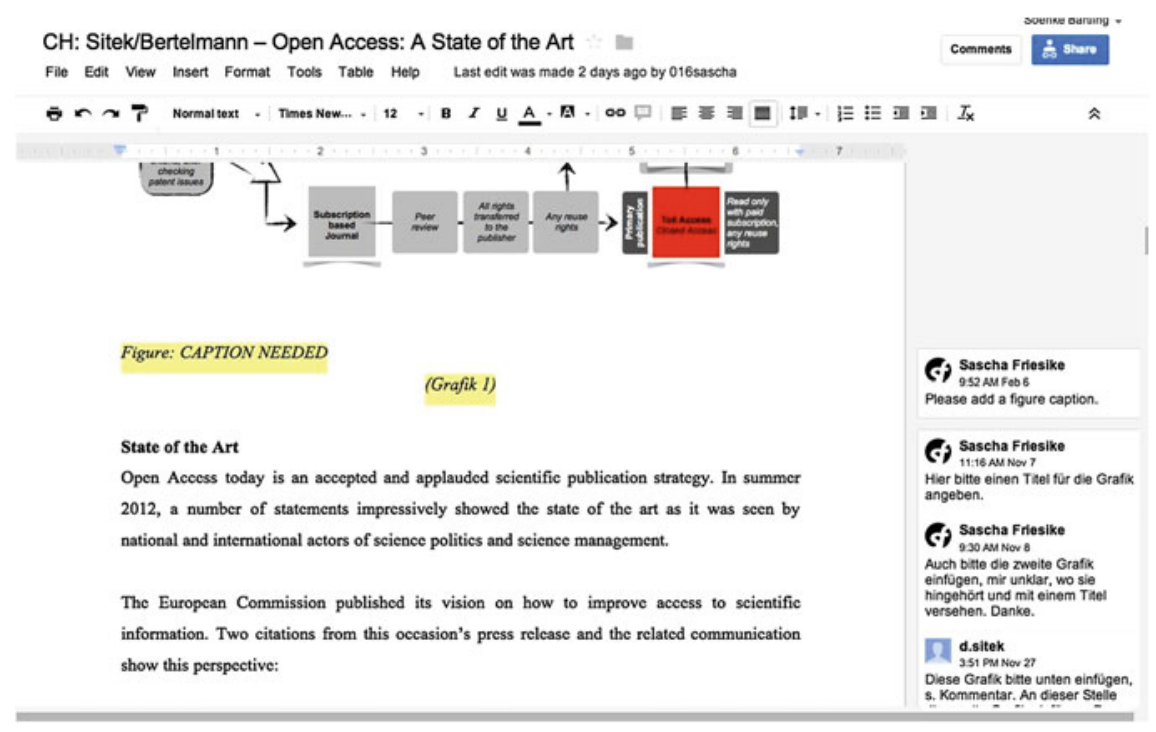

Fig. 2 During the editing process of the chapters, the authors and editors changed the chapters with editing privileges, while all others authors were invited to comment-serving as a form of internal peer-review

shared Zotero databases (one for each chapter) and manually inserted into the documents. URLs were included as footnotes.

Phase V: After reviewing changes and undertaking final proofreading, a finalized table of contents with embedded links was sent to the publisher.

Phase VI (now): The book is now available as Open Access printed book and its content can be downloaded from www.openingscience.org. Here the content can also be edited.

Open Access This chapter is distributed under the terms of the Creative Commons Attribution Noncommercial License, which permits any noncommercial use, distribution, and reproduction in any medium, provided the original author(s) and source are credited. 